\title{
Correlation of Indoleamine-2,3-Dioxygenase and Chronic Kidney Disease: A Pilot Study
}

\author{
Binbin Pan $\mathbb{D}^{1},{ }^{1}$ Feng Zhang, ${ }^{1}$ Jian Sun, ${ }^{1}$ Dawei Chen, ${ }^{1}$ Wenjuan Huang, ${ }^{1}$ Hao Zhang, ${ }^{1}$ \\ Changchun Cao, ${ }^{2}$ and Xin Wan $\mathbb{D i D}^{1}$ \\ ${ }^{1}$ Department of Nephrology, Nanjing First Hospital, Nanjing Medical University, Nanjing, China \\ ${ }^{2}$ Department of Nephrology, Sir Run Run Hospital, Nanjing Medical University, Nanjing, China \\ Correspondence should be addressed to Xin Wan; wanxin@njmu.edu.cn
}

Received 2 August 2020; Revised 28 November 2020; Accepted 19 December 2020; Published 6 January 2021

Academic Editor: Amar Singh

Copyright (c) 2021 Binbin Pan et al. This is an open access article distributed under the Creative Commons Attribution License, which permits unrestricted use, distribution, and reproduction in any medium, provided the original work is properly cited.

\begin{abstract}
Objective. To explore the correlation of indoleamine-2,3-dioxygenase (IDO) and chronic kidney disease (CKD). Methods. A total of 154 CKD patients and 42 non-CKD patients were recruited. Patients were grouped into ACR1 ACR3 ( $<30 \mathrm{mg} / \mathrm{g}, 30-300 \mathrm{mg} / \mathrm{g}$, and $>300 \mathrm{mg} / \mathrm{g}$ ). Biomarkers in different groups were compared by ANOVA. Correlation was calculated by Pearson or Spearman analysis and binary logistic regression. The ROC curve was also performed. Results. The levels of albumin, serum creatinine $(\mathrm{sCr})$, and IDO in non-CKD patients were significantly different from those in CKD3-CKD5 stages $(p<0.05)$. IDO was correlated with age, proteinuria, ACR, and eGFR $(p<0.01)$. After adjusting for CKD-related indices, $\ln ($ IDO) was an independent risk factor for CKD $(3.48, p<0.05)$. The analysis of ROC curve revealed a best cut-off for IDO was 0.0466 and yielded a sensitivity of $83.8 \%$ and a specificity of $75 \%$. Hemoglobin, total protein, and albumin in the ACR1 group were significantly higher than those in the ACR2 and ACR3 groups $(p<0.01)$, while sCr and IDO levels were significantly lower than those in the ACR2 and ACR3 groups $(p<0.01$ or $p<0.05)$. After adjusting for CKD-related indices, $\ln$ (IDO) was still an independent risk factor for ACR (OR $=2.7, p<0.05)$. The analysis of ROC curve revealed a best cut-off for IDO was 0.075 and yielded a sensitivity of $71.9 \%$ and a specificity of $72.2 \%$. Conclusion. IDO may be a promising biomarker to predict CKD and assess kidney function.
\end{abstract}

\section{Introduction}

Chronic kidney disease (CKD) is a worldwide epidemic disease with more than $10.8 \%$ prevalence in China [1]. The final common pathology of CKD is kidney fibrosis $[2,3]$. The severity of CKD is in accordance with the degree of fibrosis level. However, no data to date are available to cure CKD. Therefore, early diagnosis and management of CKD is a common sense for nephrologists. Clinically, serum creatinine (sCr), cystatin $\mathrm{C}$, and $\beta 2$-microglobulin are used commonly for assessing the severity of CKD. Nevertheless, elevations of the above markers suggest that a decrease of kidney functions is rather severe. Hence, exploring novel markers to assess CKD is indispensable.
Tryptophan (Trp) can be catalyzed by indoleamine-2,3dioxygenase (IDO) into kynurenine (Kyn), kynurenic acid, and quinolinic acid $[4,5]$. Therefore, IDO activity is often assessed by the ratio of Kyn and Trp [6-8]. A previous study in systemic sclerosis patients showed elevated levels of Kyn and decreased levels of Trp compared with limited cutaneous patients or healthy controls [8], suggesting increased levels of IDO. IDO was also significantly expressed in cortical and medulla tubules of the UUO mice [9] indicating IDO may be associated with kidney fibrosis. Diabetic nephropathy study also showed IDO activity was parallel with the severity of CKD and negatively correlated with estimated glomerular filtration rate (eGFR) [10]. Furthermore, induced expression of IDO could cause cell arrest in G0/G1 via Trp depletion 
TABLE 1: Clinical characteristics of CKD patients and non-CKD patients.

\begin{tabular}{|c|c|c|c|c|}
\hline & Non-CKD & CKD3 & CKD4 & CKD5 \\
\hline Gender (male/female) & $19 / 23$ & $38 / 16$ & $34 / 15$ & $25 / 17$ \\
\hline Age (years) & $62 \pm 13^{\# \boldsymbol{\Lambda}}$ & $69 \pm 16$ & $75 \pm 12$ & $71 \pm 11$ \\
\hline Uric acid $(\mu \mathrm{mol} / \mathrm{l})$ & $305 \pm 99^{* \# \boldsymbol{\Lambda}}$ & $391 \pm 95$ & $475 \pm 141$ & $412 \pm 105$ \\
\hline Albumin $(\mathrm{g} / \mathrm{l})$ & $39.9 \pm 2.9^{* \# \boldsymbol{\Lambda}}$ & $36.1 \pm 5.8$ & $35.5 \pm 5.0$ & $32.9 \pm 4.8$ \\
\hline Prealbumin (mg/l) & $225.1 \pm 53.5$ & $229.8 \pm 79.7$ & $222.3 \pm 68.7$ & $232.3 \pm 72.5$ \\
\hline FT3 (pmol/l) & $4.1 \pm 0.9^{\# \boldsymbol{\Lambda}}$ & $3.7 \pm 0.8$ & $3.4 \pm 0.7$ & $3.3 \pm 1.1$ \\
\hline FT4 (pmol/l) & $13.2 \pm 2.6$ & $12.6 \pm 1.9$ & $13.0 \pm 2.3$ & $12.3 \pm 2.2$ \\
\hline TSH (mIU/l) & $2.2 \pm 1.1$ & $2.0 \pm 1.3$ & $2.3 \pm 1.4$ & $2.7 \pm 1.9$ \\
\hline Complement C3 (mg/l) & $0.87 \pm 0.18^{*}$ & $0.70 \pm 0.19$ & $0.79 \pm 0.17$ & $0.71 \pm 0.19$ \\
\hline Complement C4 (mg/l) & $0.20 \pm 0.06$ & $0.25 \pm 0.18$ & $0.21 \pm 0.06$ & $0.24 \pm 0.08$ \\
\hline Hemoglobin (g/l) & $130 \pm 16^{\# \boldsymbol{\Lambda}}$ & $122 \pm 20$ & $109 \pm 25$ & $89 \pm 15^{*}$ \\
\hline Cystatin C (mg/dl) & $1.19 \pm 0.36^{* \# \boldsymbol{\Lambda}}$ & $1.75 \pm 0.40$ & $2.65 \pm 0.61$ & $3.95 \pm 0.71^{*}$ \\
\hline$\beta 2-\mathrm{MG}(\mathrm{mg} / \mathrm{dl})$ & $2.55 \pm 1.10^{* \# \boldsymbol{\triangle}}$ & $4.08 \pm 1.63$ & $7.32 \pm 2.79$ & $12.76 \pm 4.33^{*}$ \\
\hline BUN (mmol/l) & $5.71 \pm 1.73^{* \# \boldsymbol{\triangle}}$ & $8.43 \pm 2.83$ & $14.09 \pm 7.42$ & $21.92 \pm 5.30^{*}$ \\
\hline $\mathrm{sCr}(\mu \mathrm{mol} / \mathrm{l})$ & $67 \pm 14^{* \# \boldsymbol{\Lambda}}$ & $120 \pm 21$ & $191 \pm 37$ & $471 \pm 152^{*}$ \\
\hline Proteinuria $(\mathrm{g} / 24 \mathrm{~h})$ & $36 \pm 47^{* \# \boldsymbol{\Lambda}}$ & $555 \pm 1124$ & $767 \pm 976$ & $1582 \pm 1918$ \\
\hline Calcium (mmol/l) & $2.24 \pm 0.13^{* \# \boldsymbol{\Lambda}}$ & $2.14 \pm 0.15$ & $2.17 \pm 0.13$ & $2.03 \pm 0.21$ \\
\hline Phosphorus (mmol/l) & $1.18 \pm 0.19^{\# \boldsymbol{\Lambda}}$ & $1.16 \pm 0.17$ & $1.30 \pm 0.23$ & $1.52 \pm 0.36^{*}$ \\
\hline $\mathrm{TC}(\mathrm{mmol} / \mathrm{l})$ & $4.35 \pm 1.01$ & $4.48 \pm 1.63$ & $4.06 \pm 1.16$ & $3.97 \pm 0.97$ \\
\hline $\mathrm{TG}(\mathrm{mmol} / \mathrm{l})$ & $1.87 \pm 0.89^{\wedge}$ & $1.61 \pm 0.96$ & $1.58 \pm 0.71$ & $1.38 \pm 0.93$ \\
\hline HDL (mmol/l) & $1.11 \pm 0.29$ & $1.00 \pm 0.28$ & $0.96 \pm 0.24$ & $1.03 \pm 0.51$ \\
\hline $\mathrm{LDL}(\mathrm{mmol} / \mathrm{l})$ & $2.25 \pm 0.64$ & $2.53 \pm 1.25$ & $2.24 \pm 0.82$ & $2.20 \pm 0.77$ \\
\hline HbAlc (\%) & $7.28 \pm 1.71$ & $6.86 \pm 1.14$ & $6.45 \pm 0.86$ & $6.19 \pm 0.92$ \\
\hline $\operatorname{IgA}(\mathrm{mg} / \mathrm{l})$ & $2.25 \pm 0.89$ & $2.53 \pm 2.05$ & $2.58 \pm 1.36$ & $2.34 \pm 0.92$ \\
\hline $\operatorname{IgG}(\mathrm{mg} / \mathrm{l})$ & $10.23 \pm 1.96$ & $11.39 \pm 4.98$ & $12.15 \pm 3.40$ & $11.81 \pm 4.76$ \\
\hline $\operatorname{IgM}(\mathrm{mg} / \mathrm{l})$ & $1.04 \pm 0.53$ & $0.89 \pm 0.68$ & $0.87 \pm 0.46$ & $0.96 \pm 1.05$ \\
\hline Tryptophan $(\mu \mathrm{mol} / \mathrm{l})$ & $57.3 \pm 21.7^{\wedge}$ & $55.9 \pm 21.4$ & $48.3 \pm 21.6$ & $35.3 \pm 17.1^{*}$ \\
\hline Kynurenine $(\mu \mathrm{mol} / \mathrm{l})$ & $2.15 \pm 0.82^{* \# \boldsymbol{\Lambda}}$ & $3.30 \pm 1.38$ & $4.23 \pm 2.02$ & $5.34 \pm 2.73$ \\
\hline IDO & $0.04 \pm 0.02^{* \# \boldsymbol{\Lambda}}$ & $0.07 \pm 0.04$ & $0.10 \pm 0.06$ & $0.16 \pm 0.08^{*}$ \\
\hline CRP (mg/l) & $9 \pm 16$ & $12 \pm 29$ & $27 \pm 38$ & $19 \pm 35$ \\
\hline PT (second) & $11.21 \pm 1.00$ & $11.16 \pm 1.12$ & $11.79 \pm 1.43$ & $12.30 \pm 2.79$ \\
\hline APTT (second) & $26.54 \pm 2.20$ & $27.11 \pm 2.89$ & $27.22 \pm 3.47$ & $30.71 \pm 10.54$ \\
\hline Fibrinogen $(\mathrm{g} / \mathrm{l})$ & $3.35 \pm 1.04$ & $3.22 \pm 0.72$ & $3.92 \pm 1.32$ & $4.97 \pm 3.82$ \\
\hline $\mathrm{DD}(\mu \mathrm{g} / \mathrm{ml})$ & $0.57 \pm 0.48$ & $1.30 \pm 2.61$ & $2.12 \pm 5.08$ & $1.14 \pm 0.82$ \\
\hline
\end{tabular}

CKD: chronic kidney disease; FT3: free triiodothyronine; FT4: free thyroxine; TSH: thyroid-stimulating hormone; $\beta 2$-MG: $\beta 2$-microglobulin; BUN: blood urea nitrogen; sCr: serum creatinine; TC: total cholesterol; TG: triglyceride; HDL: high-density lipoprotein; LDL: low-density lipoprotein; IgA: immunoglobulin A; IgG: immunoglobulin G; IgM: immunoglobulin M; IDO: indoleamine-2,3-dioxygenase; CRP: C-reactive protein; PT: prothrombin time; APTT: activated partial thromboplastin time; DD: D-dimer. ${ }^{*} p$, non-CKD vs. CKD3; ${ }^{*} p$, non-CKD vs. CKD4; ${ }^{\boldsymbol{\Delta}} p$, non-CKD vs. CKD5; ${ }^{* \# \boldsymbol{\Lambda}} p<0.05$.

resulting in cardiac fibrosis which could be ameliorated by IDO inhibitor, 1-methyltryptophan [11]. Therefore, IDO is a potential biomarker for assessing tissue fibrosis and CKD.

Although previous studies have focused on the correlation between kidney function and IDO, little is known about the predicting function of IDO on the occurrence of CKD. In the present study, we explored the correlation of IDO with
CKD-related indices and the predictive ability of IDO on $\mathrm{CKD}$ and albuminuria and creatinine ratio (ACR).

\section{Materials and Methods}

A total of 196 patients were recruited from December 2018 to May 2020 in Nanjing First Hospital. We excluded subjects 
who had the following characteristics: younger than 18 years old, fever, pregnancy, heart failure, liver dysfunction, diabetic ketoacidosis, nonketotic hyperosmolar coma, diabetic lactic acidosis, taking $\alpha$-ketoacid in the past four weeks, and acute kidney injury.

2.1. Laboratory Measure. Cystatin C, sCr, blood urea nitrogen (BUN), uric acid, $\beta 2$-microglobin, serum albumin, calcium, phosphorus, serum lipid, and proteinuria were determined by the OLYMPUS AU5400 automatic biochemical analyzer (Olympus Corporation, Mishima, Japan). The calibrators for the enzymatic method were traceable to an isotope dilution mass spectrometric method for $\mathrm{sCr}$ using standard reference methods NIST SRM 967 [12]. ACR and $\mathrm{D}$-dimer were determined via immunoturbidimetry. Plasma prothrombin time, activated partial prothrombin time, and plasma fibrinogen were measured by the coagulation method. Thyroid function was tested by using an electrochemiluminescence assay (Siemens, Centaur XP). Hemoglobin was measured by the Sysmex XT-1800i Automated Hematology System (Shanghai, China). The values of serum immunoglobulin and complements were tested by single immunodiffusion. C-reactive protein (CRP) was tested by electrochemiluminescence. Trp and Kyn of blood samples were determined by high-performance liquid chromatography-mass spectrometry. The level of IDO was calculated by the ratio of Kyn and Trp.

2.2. Definition of CKD. Different CKD stages were divided following the KIDGO guideline: stage 1, eGFR $\geq 90$ $\mathrm{ml} / \mathrm{min} / 1.73 \mathrm{~m}^{2}$; stage $2,60 \mathrm{ml} / \mathrm{min} / 1.73 \mathrm{~m}^{2} \leq \mathrm{eGFR}<90 \mathrm{ml}$ $/ \mathrm{min} / 1.73 \mathrm{~m}^{2}$; stage $3,30 \mathrm{ml} / \mathrm{min} / 1.73 \mathrm{~m}^{2} \leq \mathrm{eGFR}<60 \mathrm{ml} /$ $\mathrm{min} / 1.73 \mathrm{~m}^{2}$; stage $4,15 \mathrm{ml} / \mathrm{min} / 1.73 \mathrm{~m}^{2} \leq \mathrm{eGFR}<30 \mathrm{ml} /$ $\mathrm{min} / 1.73 \mathrm{~m}^{2}$; stage $5, \quad \mathrm{eGFR}<15 \mathrm{ml} / \mathrm{min} / 1.73 \mathrm{~m}^{2}$. The Chronic Kidney Disease Epidemiology Collaboration (CKD-EPI) four-level race equation was employed to calculate eGFR $[13,14]$. The specific CKD-EPI four-level race GFR estimation equation was as follows:

$$
\begin{aligned}
\mathrm{eGFR}= & \operatorname{EXP}(\operatorname{LN}(151)-0.328 * \mathrm{LN}(\mathrm{sCr} / 88.4 / 0.7) \\
& + \text { age } * \operatorname{LN}(0.993))(\text { If female and creatinine }<0.7) \\
= & \operatorname{EXP}(\operatorname{LN}(151)-1.210 * \operatorname{LN}(\mathrm{sCr} / 88.4 / 0.7) \\
& + \text { age } * \operatorname{LN}(0.993))(\text { If female and creatinine } \geq 0.7) \\
= & \operatorname{EXP}(\operatorname{LN}(149)-0.412 * \operatorname{LN}(\mathrm{sCr} / 88.4 / 0.9) \\
& + \text { age } * \operatorname{LN}(0.993))(\text { If male and creatinine }<0.9) \\
= & \operatorname{EXP}(\operatorname{LN}(149)-1.210 * \mathrm{LN}(\mathrm{sCr} / 88.4 / 0.9) \\
& + \text { age } * \operatorname{LN}(0.993))(\text { If male and creatinine } \geq 0.9) .
\end{aligned}
$$

2.3. Statistical Analysis. Statistics analysis was performed by

\begin{tabular}{|c|c|c|}
\hline & $r$ & $p$ \\
\hline eGFR & -0.8 & $<0.01^{\#}$ \\
\hline Age & 0.3 & $<0.01^{\#}$ \\
\hline Albumin & -0.6 & $<0.01^{\#}$ \\
\hline $\mathrm{Ca}$ & -0.5 & $<0.01^{\#}$ \\
\hline $\mathrm{P}$ & 0.4 & $<0.01^{\#}$ \\
\hline $\mathrm{Hb}$ & -0.6 & $<0.01^{\#}$ \\
\hline CRP & 0.3 & $<0.01^{\#}$ \\
\hline Proteinuria & 0.4 & $<0.01^{\#}$ \\
\hline ACR & 0.5 & $<0.01^{\#}$ \\
\hline PT & 0.3 & $<0.01^{\#}$ \\
\hline APTT & 0.2 & $<0.01^{\#}$ \\
\hline $\mathrm{FN}$ & 0.4 & $<0.01^{\#}$ \\
\hline DD & 0.4 & $<0.01^{\#}$ \\
\hline IgA & 0.1 & 0.1 \\
\hline IgG & 0.2 & 0.05 \\
\hline $\operatorname{IgM}$ & 0.1 & 0.5 \\
\hline Complement C3 & -0.1 & 0.3 \\
\hline Complement C4 & 0.1 & 0.2 \\
\hline $\mathrm{HP}$ & 0.2 & $<0.01^{\#}$ \\
\hline $\mathrm{DM}$ & 0.1 & 0.2 \\
\hline CAD & 0.04 & 0.6 \\
\hline CI & 0.1 & 0.4 \\
\hline Gender & 0.03 & 0.7 \\
\hline UA & 0.4 & $<0.01^{\#}$ \\
\hline TC & -0.1 & 0.19 \\
\hline LDL & -0.01 & 0.9 \\
\hline HbAlc & -0.2 & 0.1 \\
\hline
\end{tabular}
PASW 22.0 statistical software (SPSS Inc., Chicago, IL, USA). Data were expressed as mean $\pm \mathrm{SD}$. One-way ANOVA was used to compare means for continuous variables. The LSD method was used for continuous variables with homogeneous variances, and Dunnett's method was used for continuous data with uneven variances. The Pearson or
TABLE 2: Correlations of IDO and CKD-related indices.

eGFR: estimated glomerular filtration rate; Ca: serum calcium; P: serum phosphorus; Hb: hemoglobin; CRP: C-reactive protein; ACR: albuminuria and creatinine ratio; PT: prothrombin time; APTT: activated partial thromboplastin time; FN: fibrinogen; DD: D-dimer; IgA: immunoglobulin A; IgG: immunoglobulin G; IgM: immunoglobulin M; HP: hypertension; DM: diabetes mellitus; CAD: coronary artery disease; CI: cerebral infarction; UA: uric acid; TC: total cholesterol; LDL: low-density lipoprotein. ${ }^{*} p<0.01$.

Spearman correlation analysis was employed to determine the correlations between IDO and other indices. In addition, the binary logistic regression was employed to explore independent influence factors for $\mathrm{CKD}$ and ACR. The receiver operating characteristic (ROC) curve was built to evaluate the prediction of IDO on CKD and ACR. The best cutoff for ROC curve was calculated with Youden's index. $p$ value $<0.05$ was considered to be statistically significant.

\section{Results}

3.1. Clinical Characteristics of Patients (Table 1). A total of 196 patients were recruited. There were 154 patients with CKD and 42 non-CKD patients. Among them, there were 3 cases in CKD1 and 6 cases in CKD2, respectively. Therefore, the accurate data of CKD1 and CKD2 were not listed. There were no significant differences in gender, prealbumin, free 
IDO (ratio of kynurenine \& tryptophan)

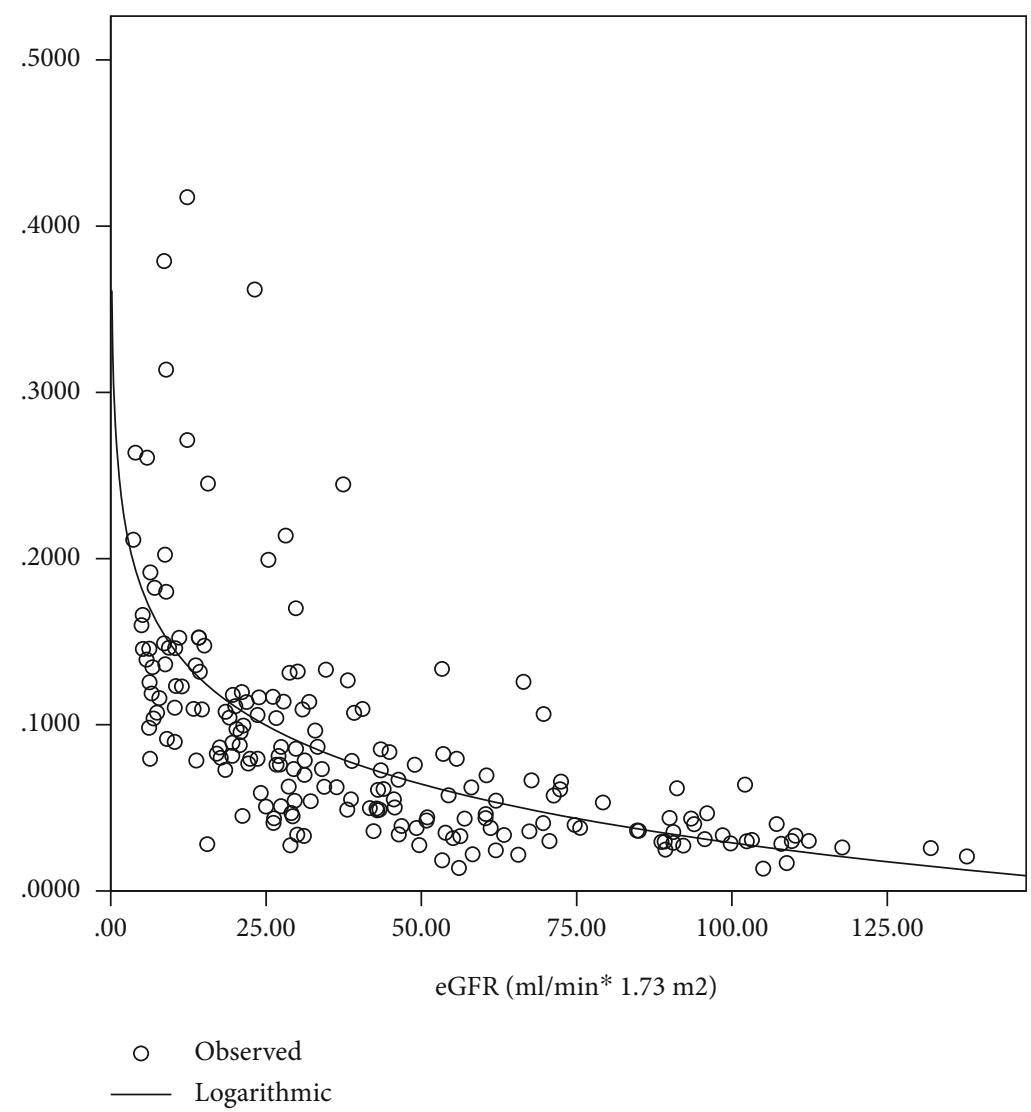

Figure 1: Estimated curve between IDO and eGFR.

TABLE 3: Binary logistic analysis of independent risk factors of chronic kidney disease.

\begin{tabular}{lcc}
\hline & $\operatorname{Exp}(\mathrm{B})(95 \% \mathrm{CI})$ & $p$ \\
\hline Age & $1.07(1.01-1.13)$ & $0.018^{*}$ \\
Hypertension & $1.14(0.32-4.04)$ & 0.85 \\
Diabetes & $3.88(0.90-16.8)$ & 0.07 \\
Coronary artery disease & $0.61(0.16-2.38)$ & 0.48 \\
Cerebral infarction & $2.47(0.55-11.1)$ & 0.24 \\
$\ln (\mathrm{IDO})$ & $3.48(1.16-10.47)$ & $0.027^{*}$ \\
Albuminuria \& creatinine ratio & $1.14(1.05-1.24)$ & $0.002^{\#}$ \\
\hline
\end{tabular}

IDO: indoleamine-2,3-dioxygenase; ${ }^{*} p<0.05,{ }^{*} p<0.01$.

thyroxine, thyroid-stimulating hormone, complement C4, total cholesterol, low-density lipoprotein, high-density lipoprotein, glycosylated hemoglobin, immunoglobulin A, immunoglobulin $\mathrm{M}$, immunoglobulin $\mathrm{G}$, and CRP among non-CKD patients and CKD3 to CKD5 patients $(p>0.05)$. The levels of uric acid, albumin, cystatin C, $\beta 2$-microglobulin, BUN, sCr, calcium, Kyn, and IDO in non-CKD patients were significantly different from those in CKD3-CKD5 stages $(p<0.05)$. There were significant differences in age, free triiodothyronine, hemoglobin, and serum phosphorus between non-CKD patients and CKD4 and CKD5 patients $(p<0.05$ ). The levels of triglyceride and Trp in non-CKD patients were significantly higher than those in CKD5 patients $(p<0.05)$.

\section{Correlations between IDO and Clinical Indices}

There was a positive correlation between IDO and age, serum phosphorus, CRP, ACR, plasma prothrombin time, activated partial prothrombin time, fibrinogen, D-dimer, hypertension, and uric acid $(r=0.3, p<0.01 ; r=0.4, p<0.01 ; r=$ $0.3, p<0.01 ; r=0.5, p<0.01 ; r=0.3, p<0.01 ; r=0.2, p<$ $0.01 ; r=0.4, p<0.01 ; r=0.4, p<0.01 ; r=0.2, p<0.01 ; r=$ $0.4, p<0.01)$. There was a negative correlation between IDO and eGFR, albumin, serum calcium, and hemoglobin $(r=-0.8, p<0.01 ; r=-0.6, p<0.01 ; r=-0.5, p<0.01 ; r=$ $-0.6, p<0.01)$ (Table 2). As IDO had a high correlation coefficient with eGFR, an estimated curve between IDO and eGFR was performed (Figure 1). As IDO is not normal distribution data, logarithm was employed to transform IDO into $\ln$ (IDO) which is normal distribution data. Further, binary logistic regression analysis was performed with CKD or not as dependent variances. The result showed that $\ln$ (IDO) was an independent risk factor for CKD. After adjusting for age, ACR, hypertension, diabetes, coronary heart disease, cerebral infarction, and $\ln (\mathrm{IDO})$, age, ACR, and $\ln (\mathrm{IDO})$ were independent risk factors for $\mathrm{CKD}(\mathrm{OR}=1.07, p<0.05$ ; $1.14, p<0.01 ; 3.48, p<0.05$ ) (Table 3). 


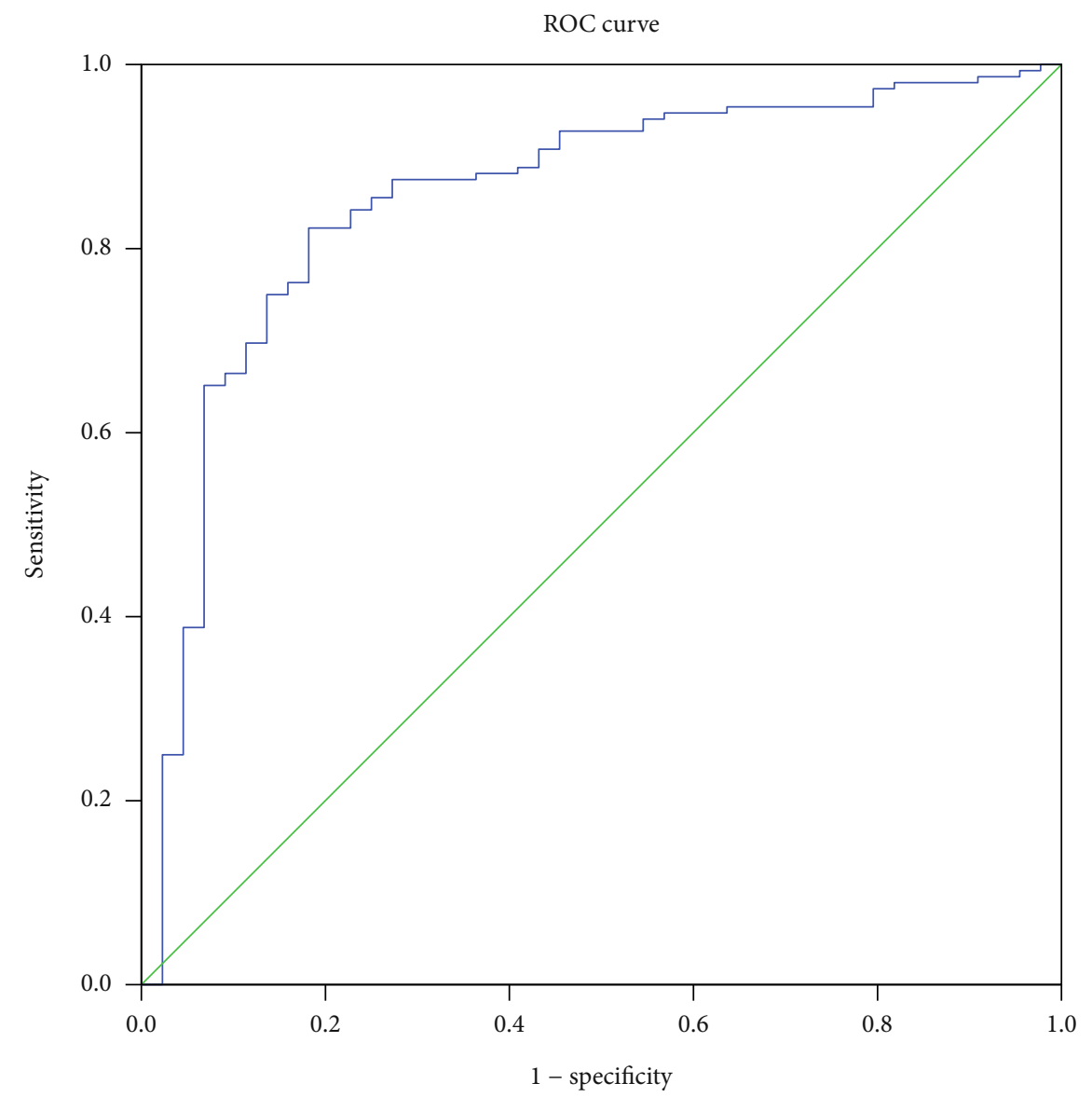

FIgURE 2: ROC curves for IDO in predicting CKD.

4.1. The Efficiency of IDO to Predict CKD. ROC curve was employed to evaluate the prediction efficiency of IDO on the CKD. The area under the ROC curve was $0.825(95 \%$ CI: $0.732-0.919, p<0.001)$ for IDO. The analysis of ROC curves revealed a best cut-off for IDO was 0.0466 and yielded a sensitivity of $83.8 \%$ and a specificity of $75 \%$ (Figure 2 ).

4.2. Correlation of IDO and ACR in CKD Patients. A total of 176 patients completed the ACR test. According to the ACR level, patients were divided into the ACR1 $(<30 \mathrm{mg} / \mathrm{g})$ group, the ACR2 (30-300 mg/g) group, and the ACR3 (>300 mg/g) group. The age of the ACR3 group was significantly lower than that of the ACR2 group $(p<0.05)$, but there was no significant difference between the ACR3 group and the ACR1 group. Hemoglobin, total protein, and albumin in the ACR1 group were significantly higher than those in the ACR2 group and the ACR3 group $(p<0.01)$, while BUN, sCr, Kyn, low-density lipoprotein, uric acid, and IDO levels were significantly lower than those in the ACR2 group and the ACR3 group $(p<0.01$ or $p<0.05)$. Fasting blood glucose $(p<0.05)$, retinol-binding protein $(p<0.01)$, and total cholesterol $(p<0.05)$ in the ACR1 group were significantly lower than those in the ACR3 group, while Trp in the ACR3 group was significantly higher than that in the ACR3 group $(p<0.01)$. Complement $\mathrm{C} 4$ and CRP were significantly lower in the ACR1 group compared with the ACR2 group $(p<0.05$
), but no significant difference was observed between the ACR3 group and the ACR1 group (Table 4).

The above correlation analysis has indicated that there was a positive correlation between ACR and IDO. Logarithm was performed to transform IDO into $\ln$ (IDO) which is normal distribution data. Binary logistic regression showed that $\ln (\mathrm{IDO})$ was an independent risk factor of ACR. After adjusting for gender, age, hemoglobin, complement C3, CRP, hypertension, diabetes, coronary artery disease, cerebral infarction, and immunoglobulin G, $\ln ($ IDO) was still an independent risk factor for ACR $(\mathrm{OR}=2.7, p<0.05)$ (Table 5).

4.3. The Efficiency of IDO to Predict ACR. ROC curve was used to evaluate the predictive effect of IDO on ACR. The area under the ROC curve of IDO was 0.753 (95\% CI: 0.68$0.826, p<0.001$ ). The analysis of ROC curves revealed a best cut-off for IDO was 0.075 and yielded a sensitivity of $71.9 \%$ and a specificity of $72.2 \%$ (Figure 3 ).

\section{Discussion}

Our study compared IDO activity calculated by the ratio of Kyn and Trp between CKD patients and non-CKD patients. The IDO activity showed an intensified trend with significant higher levels in CKD stage 4 and CKD stage 5 compared to other stages. IDO activity was also higher in elevated ACR 
TABLE 4: Comparison of clinical indices among different ACR groups.

\begin{tabular}{|c|c|c|c|}
\hline & ACR1 (<30 mg/g) & ACR2 $(30-300 \mathrm{mg} / \mathrm{g})$ & ACR3 (>300 mg/g) \\
\hline Age (years) & $68.76 \pm 14.58$ & $71.14 \pm 12.04$ & $64.38 \pm 16.72 *(3$ vs. 2$)$ \\
\hline Gender (male/female) & $47 / 33$ & $45 / 25$ & $17 / 9$ \\
\hline Hemoglobin (g/l) & $121.3 \pm 20.4^{\#}$ & $108.3 \pm 25.6$ & $102.2 \pm 27.9$ \\
\hline Total protein $(\mathrm{g} / \mathrm{l})$ & $65.0 \pm 7.29^{\#}$ & $60.1 \pm 7.05$ & $61.7 \pm 11.9$ \\
\hline Albumin $(\mathrm{g} / \mathrm{l})$ & $38.2 \pm 4.0^{\#}$ & $34.4 \pm 5.0$ & $33.0 \pm 6.9$ \\
\hline Prealbumin (mg/l) & $224.2 \pm 70.6$ & $227.6 \pm 64.3$ & $244.8 \pm 75.3$ \\
\hline Cystatin C (mg/dl) & $4.17 \pm 1.13$ & $2.87 \pm 1.05$ & $3.09 \pm 1.24$ \\
\hline $\mathrm{BUN}(\mathrm{mmol} / \mathrm{l})$ & $8.46 \pm 4.59^{\#}$ & $15.2 \pm 8.27$ & $15.6 \pm 8.8$ \\
\hline $\mathrm{sCr}(\mu \mathrm{mol} / \mathrm{l})$ & $124.9 \pm 109.4^{\#}$ & $247.4 \pm 159.3$ & $309.9 \pm 207.8$ \\
\hline $\mathrm{FBG}(\mathrm{mmol} / \mathrm{l})$ & $5.84 \pm 2.33^{*}(3$ vs. 1$)$ & $5.48 \pm 2.26$ & $4.83 \pm 1.47$ \\
\hline Uric acid $(\mu \mathrm{mol} / \mathrm{l})$ & $370.6 \pm 132^{*}$ & $425.2 \pm 123.5$ & $406.1 \pm 106.6$ \\
\hline $\mathrm{RBP}(\mathrm{mg} / \mathrm{l})$ & $20.6 \pm 31.9^{\#}$ (3 vs. 1 ) & $43.8 \pm 43.3$ & $89.3 \pm 36.0$ \\
\hline $\mathrm{TC}(\mathrm{mmol} / \mathrm{l})$ & $4.04 \pm 0.97^{*}(3$ vs. 1$)$ & $4.36 \pm 1.37$ & $4.65 \pm 1.63$ \\
\hline $\mathrm{TG}(\mathrm{mmol} / \mathrm{l})$ & $1.84 \pm 1.56$ & $1.59 \pm 0.85$ & $1.55 \pm 0.76$ \\
\hline $\mathrm{LDL}(\mathrm{mmol} / \mathrm{l})$ & $2.1 \pm 0.64^{*}$ & $2.49 \pm 1.01$ & $2.63 \pm 1.27$ \\
\hline $\mathrm{HDL}(\mathrm{mmol} / \mathrm{l})$ & $1.05 \pm 0.3$ & $0.97 \pm 0.25$ & $1.1 \pm 0.34$ \\
\hline HbAlc (\%) & $11.17 \pm 30.7$ & $6.72 \pm 1.06$ & $6.66 \pm 0.82$ \\
\hline $\operatorname{IgG}(\mathrm{mg} / \mathrm{l})$ & $12.25 \pm 3.11$ & $10.92 \pm 3.90$ & $12.56 \pm 7.05$ \\
\hline $\operatorname{IgA}(\mathrm{mg} / \mathrm{l})$ & $2.40 \pm 1.43$ & $3.86 \pm 1.23$ & $2.68 \pm 1.97$ \\
\hline $\operatorname{IgM}(\mathrm{mg} / \mathrm{l})$ & $0.92 \pm 0.57$ & $1.25 \pm 2.06$ & $0.97 \pm 0.78$ \\
\hline Complement C3 (mg/l) & $0.77 \pm 0.16$ & $0.74 \pm 0.19$ & $0.71 \pm 0.23$ \\
\hline Complement C4 (mg/l) & $0.20 \pm 0.06^{*}(2$ vs. 1$)$ & $0.25 \pm 0.15$ & $0.23 \pm 0.11$ \\
\hline CRP (mg/l) & $9.26 \pm 12.94^{*}(2$ vs. 1$)$ & $23.73 \pm 40.79$ & $22.12 \pm 40.33$ \\
\hline Tryptophan $(\mu \mathrm{mol} / \mathrm{l})$ & $54.30 \pm 20.23^{\#}(3$ vs. 1$)$ & $49.00 \pm 23.67$ & $43.36 \pm 23.73$ \\
\hline Kynurenine $(\mu \mathrm{mol} / \mathrm{l})$ & $3.00 \pm 1.42^{\#}$ & $4.46 \pm 2.45$ & $4.50 \pm 2.17$ \\
\hline IDO & $0.072 \pm 0.092^{*}$ & $0.107 \pm 0.071$ & $0.126 \pm 0.075$ \\
\hline
\end{tabular}

ACR: albuminuria and creatinine ratio; BUN: blood urea nitrogen; sCr: serum creatinine; FBG: fast blood glucose; RBP: retinol-binding protein; TC: total cholesterol; TG: triglyceride; LDL: low-density lipoprotein; HDL: high-density lipoprotein; IgG: immunoglobulin G; IgA: immunoglobulin A; IgM: immunoglobulin M; CRP: C-reactive protein; IDO: indoleamine-2,3-dioxygenase. ${ }^{*} p<0.05,{ }^{\sharp} p<0.01$.

TABLE 5: Binary logistic analysis of independent risk factors of ACR.

\begin{tabular}{lcc}
\hline & $\operatorname{Exp}(\mathrm{B})(95 \% \mathrm{CI})$ & $p$ \\
\hline Gender & $1.24(0.5-3.2)$ & 0.66 \\
Age & $0.97(0.94-1.01)$ & 0.15 \\
Hemoglobin & $0.99(0.97-1.01)$ & 0.57 \\
Complement C3 & $0.26(0.02-3.58)$ & 0.32 \\
C-reactive protein & $1.01(0.99-1.04)$ & 0.08 \\
Hypertension & $0.66(0.23-1.89)$ & 0.44 \\
Diabetes mellitus & $0.41(0.16-1.03)$ & 0.06 \\
Coronary artery disease & $1.78(0.69-4.59)$ & 0.24 \\
Cerebral infarction & $0.67(0.25-1.80)$ & 0.43 \\
ln(IDO) & $2.70(1.20-6.10)$ & $0.02 *$ \\
Immunoglobulin G & $0.90(0.81-1.01)$ & 0.07 \\
\hline
\end{tabular}

IDO: indoleamine-2,3-dioxygenase; ${ }^{*} p<0.05$. patients. Furthermore, IDO had a pretty high correlation coefficient with eGFR and ACR which were verified by the binary logistic regression analysis. On top of that, the ROC curve showed that IDO had a rather high ability to predict CKD with $83.8 \%$ in sensitivity and $75 \%$ in specificity and ACR with $71.9 \%$ in sensitivity and $72.2 \%$ in specificity suggesting IDO may be a promising biomarker for assessing CKD.

IDO is a rate-limiting enzyme of Trp which is an essential amino acid for the human body. Disorder of Trp metabolism is associated with many diseases. More than $75 \%$ of the clear cell in renal cell carcinoma patients contained elevated levels of IDO [15] which was also observed in CKD patients, and IDO activity was correlated with disease severity and levels of inflammatory markers [16]. Moreover, the increment of IDO was also observed in the crescentic glomerulonephritis model which was exacerbated after dealing with 1methyltryptophan [17] attributed to breaking down the immune tolerance induced by IDO. This kind of immune 


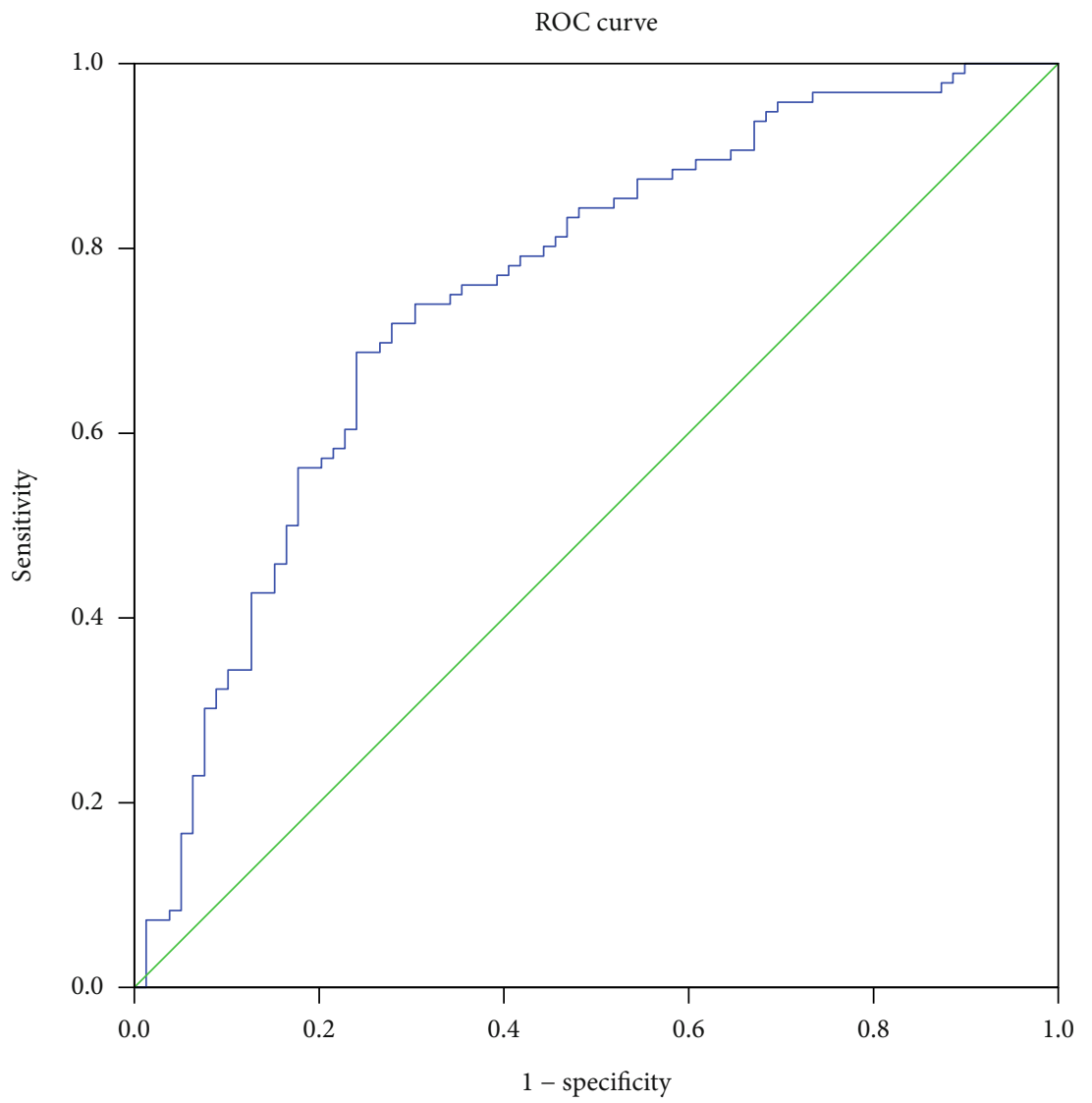

Figure 3: ROC curves for IDO in predicting ACR.

tolerance is fulfilled via differentiating $\mathrm{T}$ cells into regulatory $\mathrm{T}$ cells which plays an important role in renal allograft rejection [18-20]. Patients with acute kidney allograft rejection revealed higher expressions of IDO compared with nonrejectors [19]. Although the previous study has proved the IDO activity was an independent risk factor of sCr [16], the accurate correlation between IDO and kidney function is largely unknown. IDO has been suggested to be associated with fibrosis which may be controversial in different fields. In the field of a liver fibrosis model induced by $\mathrm{CCl} 4$, mesenchymal stem cells could attenuate liver fibrosis by increasing IDO [19] and deficiency of IDO could enhance the inflammation in the liver and aggravated liver fibrosis [21]. Further, IDO knockout mice also showed more susceptible to high fat diet-induced hepatic inflammation and fibrosis [22]. Another study showed that using 1-methyltryptophan to inhibit IDO could enhance the suppression of activated hepatic stellate cells which mediates the extracellular matrix deposition in the liver to promote fibrosis [23]. Fibrosis in cardiac tissue was also induced by overexpression of IDO [11] via cell arrest. Mutations in the cystic fibrosis have been reported to have a defect in IDO expression and resulted in intensified fibrosis [6]. On the contrary, kidney studies revealed elevated expression of IDO in tubules in the unilateral ureteral obstruction model and transforming growth factor- $\beta$ stimulated MDCK cells [9] as well as diabetic nephropathy patients [10]. Our study also revealed increased activity of IDO in CKD patients, especially in CKD stage 5.
In our study, the correlation coefficient between eGFR and IDO was 0.8 , which was higher than that in a previous study [16]. Further, binary logistic regression showed age, IDO, and ACR were independent risk factors for CKD. As is known that age and proteinuria are independent risk factors for CKD [24], while IDO was a novel risk factor of CKD. In addition, previous studies in diabetic patients [16] and obstructive nephropathy [12] have confirmed that IDO is associated with eGFR. However, whether IDO can be used to evaluate the development and progression of CKD still needs a large scale of clinical studies to provide more conclusive evidence.

Renin-angiotensin-aldosterone system inhibitors have been reported to inhibit the activity of Kyn which has a positive correlation with albuminuria [25]. However, this study did not explore the relationship between IDO and albuminuria. In pregnant patients, lower IDO expression level was associated with more severe pregnancy-related hypertension and proteinuria [26]. Therefore, the relationship between IDO and proteinuria is still controversial. Our results suggest that IDO is positively correlated with proteinuria, suggesting that disorder of IDO activity may increase proteinuria. Further binary regression analysis confirmed that IDO was an independent factor of ACR.

In this study, IDO was also found to be associated with other CKD-related indicators, such as calcium, phosphorus, uric acid, hemoglobin, albumin, coagulation indicators, and hypertension. CKD patients, especially those with CKD3 or 
server stage are commonly accompanying with low calcium, high phosphorus, hyperuricemia, renal anemia, and protein malnutrition as well as increased prevalence of hypertension [27]. Meanwhile, the level of IDO was increased with the increment of the CKD stage. Therefore, correlations between IDO and the above indicators may be caused by CKD itself. The correlation between IDO and coagulation parameters has not been reported before. CKD leads to the accumulation of uremic toxin, which has a toxic effect on blood and vascular wall. Some uremic toxins can be metabolized in the intestinal tract through indole and Kyn pathways. Studies have confirmed that these uremic toxins may be associated with thrombosis in chronic kidney disease by increasing plasma procoagulant factor levels, hyperactivity of platelets, impaired endothelial dysfunction/endothelial healing, decreased nitric oxide bioavailability, and production of procoagulant particles [28]. Therefore, IDO may indirectly affect the coagulation function of CKD patients to promote thrombosis. The specific mechanism needs further research. The correlation between IDO and CRP seems to be similar to previous studies [29], suggesting that IDO may be involved in the chronic inflammatory state of CKD; however, in our study, there was no significant difference in CRP levels among different groups, suggesting that IDO does not promote the progress of CKD by increasing chronic inflammatory level.

There are some limitations in the present study. Firstly, we did not recruit enough CKD stage 1 and CKD stage 2 patients to observe the level of IDO in these two stages which was the same as a previous study [16]. It could be useful to study the levels of IDO in these two stages which could be better for assessing kidney function. Secondly, the sample size in our study was still not large enough although bigger than a previous study $[9,16]$. But the correlation coefficient of eGFR and IDO was pretty high, and the ROC showed that IDO was a promising biomarker for CKD enhancing the negative relationship between IDO and CKD.

Taken together, IDO was an independent influence factor of eGFR and ACR and could be used as a biomarker to assess CKD.

\section{Data Availability}

The data can be available via sending email to panbinbin@njmu.edu.cn or wanxin@njmu.edu.cn.

\section{Ethical Approval}

The study was performed in accordance with the Declaration of Helsinki and was approved by the Institutional Review Board of Nanjing First Hospital.

\section{Consent}

Informed consent was obtained from all participants when they were admitted to the hospitals.

\section{Disclosure}

The funders had no role in study design, data collection and analysis, decision to publish, or reparation of the manuscript.

\section{Conflicts of Interest}

The authors have nothing to disclose.

\section{Authors' Contributions}

The research was designed by B.P. and X.W. All authors helped to write the report and commented on the manuscript. B.P. analyzed the data and advised on statistical issues at the time of the research write-up. X.W. was the research administrator, obtained the data, and prepared communications with participating centers and the data monitoring committee. D.W.C, J.S, F.Z, W.J.H., and H.Z. were research nurses responsible for recruitment and return of data.

\section{Acknowledgments}

This work was supported by grants from the Foundation of Science and Technology Development Program, Nanjing Medical University (NMUB2018321), the Young Medical Talents Program of Jiangsu (QNRC2016067) and the National Science Foundation of China (81670634).

\section{References}

[1] L. Zhang, F. Wang, L. Wang et al., "Prevalence of chronic kidney disease in China: a cross-sectional survey," The Lancet, vol. 379 , no. 9818 , pp. 815-822, 2012.

[2] M. V. Nastase, J. Zeng-Brouwers, M. Wygrecka, and L. Schaefer, "Targeting renal fibrosis: mechanisms and drug delivery systems," Advanced Drug Delivery Reviews, vol. 129, pp. 295-307, 2018.

[3] A. C. Webster, E. V. Nagler, R. L. Morton, and P. Masson, "Chronic kidney disease," The Lancet, vol. 389, no. 10075, pp. 1238-1252, 2017.

[4] T. W. Stone and L. G. Darlington, "Endogenous kynurenines as targets for drug discovery and development," Nature Reviews. Drug Discovery, vol. 1, no. 8, pp. 609-620, 2002.

[5] A. A. Badawy, "Targeting tryptophan availability to tumors: the answer to immune escape?," Immunology and Cell Biology, vol. 96, no. 10, pp. 1026-1034, 2018.

[6] R. G. Iannitti, A. Carvalho, C. Cunha et al., "Th17/Treg imbalance in murine cystic fibrosis is linked to indoleamine 2,3dioxygenase deficiency but corrected by kynurenines," American Journal of Respiratory and Critical Care Medicine, vol. 187, no. 6, pp. 609-620, 2013.

[7] M. Toulmonde, N. Penel, J. Adam et al., "Use of PD-1 targeting, macrophage infiltration, and IDO pathway activation in sarcomas: a phase 2 clinical trial," JAMA Oncology, vol. 4, no. 1, pp. 93-97, 2018.

[8] C. Campochiaro, S. Lytton, S. Nihtyanova, D. Fuchs, V. H. Ong, and C. P. Denton, "Elevated kynurenine levels in diffuse cutaneous and anti-RNA polymerase III positive systemic sclerosis," Clinical Immunology, vol. 199, pp. 18-24, 2019.

[9] L. H. G. Matheus, G. M. Simão, T. A. Amaral et al., "Indoleamine 2, 3-dioxygenase (IDO) increases during renal 
fibrogenesis and its inhibition potentiates TGF- $\beta$ 1-induced epithelial to mesenchymal transition," BMC Nephrology, vol. 18, no. 1, p. 287, 2017.

[10] Y. Zhang, Y. Ruan, P. Zhang, and L. Wang, "Increased indoleamine 2,3-dioxygenase activity in type 2 diabetic nephropathy," Journal of Diabetes and its Complications, vol. 31, no. 1, pp. 223-227, 2017.

[11] J. W. Lee, J. E. Oh, K. J. Rhee et al., "Co-treatment with interferon- $\gamma$ and 1-methyl tryptophan ameliorates cardiac fibrosis through cardiac myofibroblasts apoptosis," Molecular and Cellular Biochemistry, vol. 458, no. 1-2, pp. 197-205, 2019.

[12] N. G. Dodder, S. S. Tai, L. T. Sniegoski, N. F. Zhang, and M. J. Welch, "Certification of creatinine in a human serum reference material by GC-MS and LC-MS," Clinical Chemistry, vol. 53, no. 9, pp. 1694-1699, 2007.

[13] X. Du, L. Liu, B. Hu et al., "Is the Chronic Kidney Disease Epidemiology Collaboration fourlevel race equation better than the cystatin C equation?," Nephrology (Carlton, Vic.), vol. 17, no. 4, pp. 407-414, 2012.

[14] L. A. Stevens, M. A. Claybon, C. H. Schmid et al., "Evaluation of the Chronic Kidney Disease Epidemiology Collaboration equation for estimating the glomerular filtration rate in multiple ethnicities," Kidney International, vol. 79, no. 5, pp. 555562, 2011.

[15] R. Riesenberg, C. Weiler, O. Spring et al., "Expression of indoleamine 2,3-dioxygenase in tumor endothelial cells correlates with long-term survival of patients with renal cell carcinoma," Clinical Cancer Research, vol. 13, no. 23, pp. 6993-7002, 2007.

[16] J. C. Schefold, J. P. Zeden, C. Fotopoulou et al., "Increased indoleamine 2,3-dioxygenase (IDO) activity and elevated serum levels of tryptophan catabolites in patients with chronic kidney disease: a possible link between chronic inflammation and uraemic symptoms," Nephrology, Dialysis, Transplantation, vol. 24, no. 6, pp. 1901-1908, 2009.

[17] W. Hou, S. Li, Y. Wu, X. Du, and F. Yuan, "Inhibition of indoleamine 2, 3-dioxygenase-mediated tryptophan catabolism accelerates crescentic glomerulonephritis," Clinical and Experimental Immunology, vol. 156, no. 2, pp. 363-372, 2009.

[18] D. Vavrincova-Yaghi, L. E. Deelman, H. van Goor et al., "Local gene therapy with indoleamine 2,3-dioxygenase protects against development of transplant vasculopathy in chronic kidney transplant dysfunction," Gene Therapy, vol. 23, no. 11, pp. 797-806, 2016.

[19] G. Brandacher, F. Cakar, C. Winkler et al., "Non-invasive monitoring of kidney allograft rejection through IDO metabolism evaluation," Kidney International, vol. 71, no. 1, pp. 60-67, 2007.

[20] D. Vavrincova-Yaghi, M. A. Seelen, I. P. Kema et al., "Early posttransplant tryptophan metabolism predicts long-term outcome of human kidney transplantation," Transplantation, vol. 99, no. 8, pp. e97-e104, 2015.

[21] H. Ogiso, H. Ito, T. Ando et al., "The deficiency of indoleamine 2,3-dioxygenase aggravates the CCl4-induced liver fibrosis in mice," PLoS One, vol. 11, no. 9, article e0162183, 2016.

[22] J. Nagano, M. Shimizu, T. Hara et al., "Effects of indoleamine 2,3-dioxygenase deficiency on high-fat diet-induced hepatic inflammation," PLoS One, vol. 8, no. 9, article e73404, 2013.

[23] J. E. Oh, K. Y. Shim, J. I. Lee, S. I. Choi, S. K. Baik, and Y. W. Eom, "1-Methyl-L-tryptophan promotes the apoptosis of hepatic stellate cells arrested by interferon $-\gamma$ by increasing the expression of IFN- $\gamma \mathrm{R} \beta$, IRF- 1 and FAS," International
Journal of Molecular Medicine, vol. 40, no. 2, pp. 576-582, 2017.

[24] M.-Y. Lin, Y.-W. Chiu, C.-H. Lee et al., "Factors associated with CKD in the elderly and nonelderly population," Clinical Journal of the American Society of Nephrology, vol. 8, no. 1, pp. 33-40, 2013.

[25] V. Cernaro, S. Loddo, V. Macaione et al., "RAS inhibition modulates kynurenine levels in a CKD population with and without type 2 diabetes mellitus," International Urology and Nephrology, vol. 52, no. 6, pp. 1125-1133, 2020.

[26] N. Iwahashi, M. Yamamoto, S. Nanjo, S. Toujima, S. Minami, and K. Ino, "Downregulation of indoleamine 2, 3-dioxygenase expression in the villous stromal endothelial cells of placentas with preeclampsia," Journal of Reproductive Immunology, vol. 119, pp. 54-60, 2017.

[27] K. Elaine, B. J. Lee, J. Wei, and M. R. Weir, "Hypertension in CKD: core curriculum 2019," American Journal of Kidney Diseases, vol. 74, no. 1, pp. 120-131, 2019.

[28] T. Addi, L. Dou, and S. Burtey, "Tryptophan-derived uremic toxins and thrombosis in chronic kidney disease," Toxins (Basel), vol. 10, no. 10, p. 412, 2018.

[29] M. Pertovaara, A. Raitala, M. Juonala et al., "Indoleamine 2,3dioxygenase enzyme activity correlates with risk factors for atherosclerosis: the Cardiovascular Risk in Young Finns Study," Clinical and Experimental Immunology, vol. 148, no. 1, pp. 106-111, 2007. 